\title{
The Use of Low-Dose Methadone as Add-On to Regular Opioid Therapy in Cancer-Related Pain at End of Life: A National Swedish Survey in Specialized Palliative Care
}

\author{
Per Fürst, MD, ${ }^{1,2}$ Staffan Lundström, MD, PhD, ${ }^{1,2}$ Pål Klepstad, MD, PhD, ${ }^{3-5}$ and Peter Strang, MD, PhD ${ }^{1,2}$
}

\begin{abstract}
Background: Low-dose methadone in addition to another ongoing opioid therapy is a promising approach for managing complex cancer-related pain and is, despite limited evidence, used in clinical practice.

Objective: To investigate the use of low-dose methadone in specialized palliative care in Sweden.

Design: Specialized palliative care services in Sweden answered a survey regarding methadone use in individual patients over 12 months.

Setting/Subjects: The survey was an add-on to the Swedish Register of Palliative Care's (SRPC) mandatory end-of-life questionnaire (ELQ).

Results: Sixty of 133 invited units (45\%) participated in the study. A total of 4780 ELQs were registered. Four hundred ten of these patients received methadone (9\%). In $96 \%$ of these patients, methadone was prescribed as an add-on to ongoing opioid therapy, mostly because of poor pain control due to mixed nociceptive and neuropathic pain $(70 \%)$. Methadone was used for a median of 21 days, in $86 \%$ of cases until death. Mean daily methadone doses increased from $7 \mathrm{mg}$ at start to $21 \mathrm{mg}(p<0.005)$ during the last 24 hours. Corresponding morphine equivalent daily doses of other opioids were 184 and $199 \mathrm{mg}(p<0.05)$, respectively. A pain-relieving effect was reported in $94 \%$ of the patients. Adverse effects were seen in $20 \%$ of the patients; none of these was severe.

Conclusion: The addition of low-dose methadone to an ongoing opioid therapy in patients with complex cancerrelated pain is well established in Swedish specialized palliative care. It appears to have good pain-relieving effects and to be safe.
\end{abstract}

Keywords: add-on; cancer pain; methadone; opioid; palliative care

\section{Introduction}

C ANCER-RELATED PAIN is a frequent clinical problem in palliative care. In a Dutch study, the prevalence of severe pain in advanced cancer was reported to be as high as 66\%. ${ }^{1}$ A review from 2014 reported that approximately one third of patients with cancer-related pain still do not receive pain medication proportional to the intensity of their pain. ${ }^{2}$ Opioids are often effective as a first-line treatment in nociceptive cancer-related pain, but in complex pain situations, with a combination of nociceptive and neuropathic pain mechanisms, pain management often remains a challenge. To optimize treatment, opioids are frequently combined with tricyclic antidepressants, gabapentin, pregabalin, or serotoninnoradrenaline reuptake inhibitors, as well as with steroids and/or nonsteroidal anti-inflammatory drugs in the case of inflammatory pain components. However, an inadequate response to pharmacological treatment constitutes a substantial unmet need in patients with complex pain. ${ }^{3}$

The N-methyl-D-aspartate (NMDA) receptor pathway is of interest in complex pain as it is involved in the development of hyperalgesia, opioid tolerance, and central sensitization, resulting in reduced opioid responsiveness. Methadone is an opioid with unique analgesic properties that

\footnotetext{
${ }^{1}$ Department of Oncology-Pathology, Karolinska Institutet, Stockholm, Sweden.

${ }^{2}$ Palliative Medicine, Stockholms Sjukhem Foundation, Stockholm, Sweden.

${ }^{3}$ Department of Circulation and Medical Imaging, Faculty of Medicine, Norwegian University of Science and Technology, Trondheim, Norway.

${ }^{4}$ European Palliative Research Centre, Department of Clinical and Molecular Medicine, Faculty of Medicine, Norwegian University of Science and Technology, Trondheim, Norway.

${ }^{5}$ Department of Anesthesiology and Intensive Care medicine, St. Olavs Hospital, Trondheim University Hospital, Trondheim, Norway. Accepted July 30, 2019.
} 
stimulates regular mu-, kappa-, and delta-opioid receptors. It exerts NMDA receptor-inhibiting effects and affects the reuptake of serotonin and norepinephrine in the pain modulating descending tracts in the medulla. ${ }^{4,5}$

Despite its unique properties, many clinicians are reluctant to use methadone because of complicated pharmacokinetics and numerous drug interactions. ${ }^{6}$ Methadone has a complex pharmacokinetic profile and even low doses can result in an opioid overdose, especially in combination with high doses of other strong opioids (i.e., morphine, oxycodone, hydromorphone, or fentanyl). ${ }^{6-8}$ The guidelines from the European Association for Palliative Care (EAPC) therefore recommend that methadone should only be used by experienced professionals. ${ }^{9}$

Methadone does not produce superior analgesia when compared with morphine as a first-line opioid, ${ }^{10,11}$ especially when low doses are sufficient in opioid-responsive pain. Nonetheless, over the last 20 years, based upon methadone pharmacological properties and anecdotal evidence, there has been a rising interest among palliative care physicians for the use of methadone to treat complex pain. ${ }^{10,12-14}$ However, the actual indications and the extent of the use of methadone therapy in palliative care are unknown.

\section{$\operatorname{Aim}(s)$}

The primary objective of this study was to investigate the use of methadone for pain management in end-of-life situations in specialized palliative care settings in Sweden. Specifically, we wanted to study the frequency of use, indications, doses, opioid combinations, and observed adverse effects of low-dose methadone added to another regular opioid therapy.

\section{Methods}

\section{Study design and subjects}

In this study, the Swedish Register of Palliative Care (SRPC) was used to collect data on methadone treatment. SRPC is a national quality register that contributes to the research and development of palliative care in Sweden. The registrations are made by health care staff as soon as possible after the death of a person through an online end-of-life questionnaire (ELQ). The aim is to collect data from all deaths in Sweden, irrespective of age, diagnosis, or place of care. The ELQ provides, among other data, information regarding demographics, diagnoses, prevalence, and changes of pain intensities, breakthrough pain, anxiety, dyspnea, nausea, delirium, and death rattles during the last week of life. The ELQ also examines to what extent the outcome is based on the use of validated instruments for symptom assessments, for example, the Visual Analogue Scale (VAS) and the Numeric Rating Scale (NRS) for pain or the Edmonton Symptom Assessment Scale (ESAS) and IPOS (Integrated Palliative Care Outcome Scale) for other symptoms. The working method of the register has been described previously. ${ }^{15}$ The ELQ reflects quality of care delivered during the last week of life. In 2016, $10.8 \%$ of the 91,029 individuals who died in Sweden were enrolled in specialized palliative care and registered in the SRPC. $^{16}$

All specialized palliative care units in Sweden using the SRPC were invited to participate in this study. Participating units had an additional methadone survey added to their mandatory ELQ, which contained questions regarding the use of methadone for pain management in individual patients. Over 12 consecutive months, completion of the survey form was mandatory in cases when methadone had been initiated during a patient's care episode. The add-on survey was completed by the responsible physician and/or registered nurses.

A typical specialized palliative care team in Sweden is made up of at least one consultant and a few physicians under training, several registered district or palliative care nurses, a social worker, a physiotherapist, and an occupational therapist. The total number of physicians who are specialists in palliative medicine (a specialty since 2015) in Sweden recently reached 100 (in a population of about 10 million people). Generally, the questions included in the ELQ are discussed by the team and then reported by the physician or nurse in charge.

Analgesic and adverse effects were mainly based on patientreported outcome measures (PROMs). Based on these PROMs, pain was then assessed and reported at the referents' discretion. For example, achieved analgesia was reported on a four-graded scale as very good (complete or nearly complete analgesic effect), good (considerable analgesic effect, some remaining pain, but the patient does not request additional treatment), moderate (some analgesic effect, requires additional treatment), or of no effect. Occurrence of adverse effects was rated as present or not. A transcript of the methadone add-on survey is presented in Appendix 1. Morphine equivalent daily doses (MEDD) were calculated according to guidelines from MD Anderson Hospital (Houston, TX). ${ }^{17}$

\section{Statistics and ethics}

Descriptive statistics presenting medians with interquartile ranges and means with standard deviations were used. Percentages were used to present categorical variables. Differences in proportions were analyzed using the chi-square test and differences in ratio scales using the $t$ test or, for nonnormally distributed data, the Mann-Whitney test. The Wilcoxon signed-rank test was used to compare opioid doses from start to the final 24 hours with methadone presented as $p$-values. Analyses were performed using SPSS version 25.0.0.1.

Ethics approval (2015/1486-32) was obtained from the Regional Ethical Review Board (Stockholm, Sweden).

\section{Results}

One hundred thirty-three specialized palliative in-care and home care services were invited, of which 60 units (45\%) from all parts of Sweden participated in the study. Each unit collected data for 12 months with the first unit starting in January 2017 and the last unit finishing in June 2018. During this period, 10,058 individual ELQs were registered by specialized palliative care units, of which $4780(48 \%)$ were registered by units participating in our study. Methadone was initiated in 410 patients $(8.6 \%)$. Seventy-five percent of the ELQs and add-on surveys were completed by registered nurses and the remainder by physicians, in most cases after a team discussion.

\section{Patients}

The patients initiated on methadone had a mean age of 68 years (median 70 years), while those not treated with methadone had a mean age of 74 years (median $75 ; p<0.001$ ). In 
the methadone and nonmethadone groups, $41 \%$ and $55 \%$, respectively, were women. Eighty-seven percent of the patients on methadone had a cancer diagnosis; the corresponding number in the nonmethadone group was $82 \%$. Cardiac and respiratory diseases were less frequent in the methadone group (3\% and $1 \%$, respectively) than in the nonmethadone group (11\% and $6 \%$, respectively). Methadone was initiated at an in-care unit in $56 \%$ and at home in $36 \%$ of the patients. This corresponds to $4.8 \%$ and $3.1 \%$, respectively, of the total cohort of 4780 patients. For $8 \%$, there were missing values for the type of care setting for initiation.

\section{Indications for methadone treatment}

In most cases (96\%), methadone was used as an add-on medication to an ongoing opioid therapy. Methadone was initiated due to poor pain control in $74 \%$ of the 410 patients, of which $46 \%$ reported a pain level of $>6$ on a numeric10point rating scale. In $17 \%$ of the patients, methadone was initiated as the primary therapy against neuropathic pain, in $4 \%$ to reduce adverse effects from other opioids (e.g., attempts to reduce opioid-induced confusion and sedation), and in $5 \%$ for other reasons.

The pain mechanisms were assessed in $96 \%$ of the patients. Mixed nociceptive and neuropathic pain was the most common pain mechanism $(70 \%)$ followed by neuropathic pain $(16 \%)$, nociceptive pain $(11 \%)$, and unreported in $3 \%$.

\section{Ongoing regular opioid medication when initiating methadone treatment}

In 394 (96\%) patients, methadone was combined with another opioid (50\% fentanyl, 32\% oxycodone, $11 \%$ morphine, $6 \%$ hydromorphone, $1 \%$ ketobemidone, and $0.3 \%$ buprenorphine). The total median MEDD at initiation, excluding methadone, was $184 \mathrm{mg}$ (mean $456 \mathrm{mg}$ ) and during the last 24 hours, $199 \mathrm{mg}$ (mean $457 \mathrm{mg} ; p<0.05$ ). Two outliers with a MEDD of 4800 and 7200, respectively, were excluded from the calculation of mean doses.

\section{Methadone treatment}

For opioid doses, see Table 1. Overall, at start and during the last 24 hours, the mean methadone doses were 7 and $21 \mathrm{mg}$, respectively $(p<0.001)$. The corresponding doses for methadone used as a single opioid were $10.4-23.0 \mathrm{mg}$ $(p=0.22)$ and as a coanalgesic to other opioids were 6.9$15.5 \mathrm{mg}$ daily $(p<0.001)$.

The most commonly used initial prescriptions were $5 \mathrm{mg}$ $(52 \%)$ or $10 \mathrm{mg}(24 \%)$ per 24 hours, once $(22 \%)$ or twice $(58 \%)$ daily. Methadone doses increased during the care episode in $70 \%$ of the patients.

Methadone was used for a median of 21 days (mean 48, range 1-359). Discontinuation of methadone was due to death in $86 \%$ of the patients. Other reasons were an inability to swallow tablets $(10 \%)$, no effect of methadone $(2 \%)$, or adverse effects related to methadone (2\%).

From initiation to the final 24 hours, the proportion of patients receiving oral administration of methadone changed from $72 \%$ to $21 \%$.

\section{Analgesic effects}

VAS/NRS, ESAS or IPOS was used for assessment of pain in $84 \%$ of the patients. ${ }^{18,19}$ Methadone was reported to have a very good or good analgesic effect in $69 \%$, moderate effect in $25 \%$, and no effect in $6 \%$ of the patients.

\section{Adverse effects}

Fifty percent of patients were assessed using a validated symptom assessment tool. While $80 \%$ of the patients had no registered adverse effects, sedation and delirium were

TABLE 1. OPIOIDS

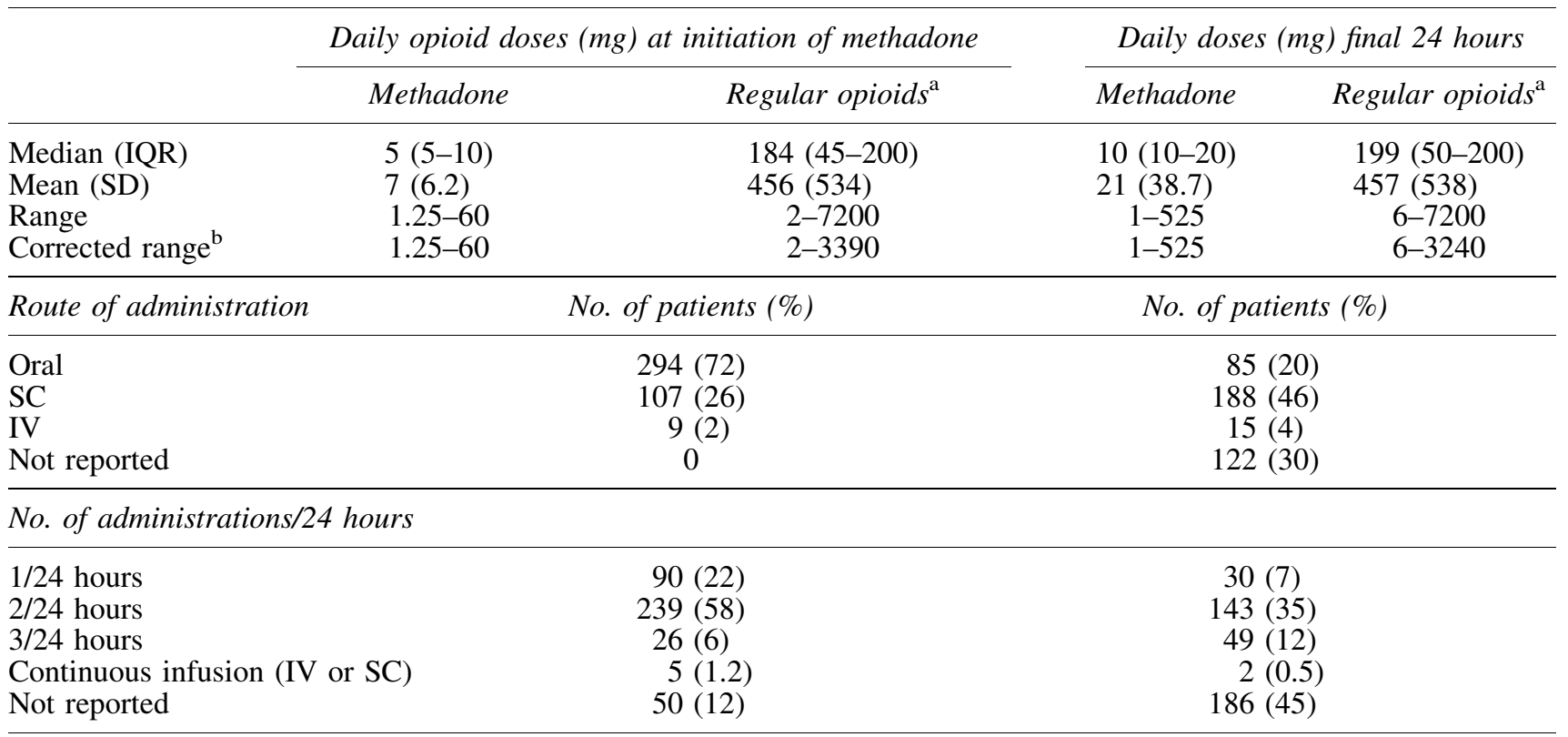

${ }^{a}$ Opioids calculated as MEDD.

${ }^{\mathrm{b}}$ Corrected ranges when excluding two outliers with doses of 4800 and 7200 MEDD, respectively.

IQR, interquartile range; IV, intravenous; MEDD, morphine equivalent daily dose; SC, subcutaneous; SD, standard deviation. 
TABle 2. Breakthrough Symptoms

\begin{tabular}{lccccc}
\hline & \multicolumn{2}{c}{ Symptom prevalence } & & \multicolumn{2}{c}{ Complete or partial symptom relief } \\
\cline { 2 - 3 } \cline { 5 - 6 } & Methadone group (\%) & Nonmethadone group (\%) & & Methadone group (\%) & Nonmethadone group (\%) \\
\hline Pain & $91^{* * *}$ & 77 & 56 & $91^{* * *}$ & 76 \\
Anxiety & $65^{* *}$ & 27 & & $65^{* *}$ & 55 \\
Delirium & $33^{* * *}$ & 27 & & $17^{* *}$ & 23 \\
Dyspnea & $20^{* * *}$ & 17 & 16 & 26 \\
Nausea & 16 & 48 & & 45 & 47 \\
Death rattles & 48 & & &
\end{tabular}

Proportion of patients who experienced episodes of increased symptoms during their last week of life, and proportion of patients who experienced complete or partial symptom relief.

Significance of difference between methadone and nonmethadone groups: ${ }^{*} p<0.05,{ }^{* *} p<0.01,{ }^{* * *} p<0.001$.

reported in $8.5 \%$ and $7.3 \%$ of the cases, respectively. Some degree of respiratory depression (not severe enough for treatment discontinuation) was registered in three hospitalized patients, but none in specialized home care. Of these three patients, one discontinued methadone because of no analgesic effect. The two others continued methadone until death, for 52 and 80 days, respectively.

During the final 24 hours, delirious and nondelirious patients had a methadone dose of median $10 \mathrm{mg}$ (mean 15.8) and $15 \mathrm{mg}$ (mean 23.8), respectively $(p=0.46)$. Corresponding figures for a total MEDD of regular opioids were median $242 \mathrm{mg}$ (mean 521) and median $195 \mathrm{mg}$ (mean 420), respectively $(p=0.49)$.

\section{Breakthrough symptoms and degree of symptom relief}

The regular ELQ enabled a comparison between the methadone and the nonmethadone groups of occurrences of breakthrough symptoms and of degree of symptom relief during the last week of life. In the methadone group, episodes of increased pain, anxiety, and delirium occurred more frequently. However, when present, pain $(p<0.001)$, anxiety $(p<0.01)$, and delirium $(p<0.05)$ were also more often relieved in the methadone group. Dyspnea was less common in patients on methadone $(p<0.001)$ (Table 2).

\section{Physicians}

Among the physicians initiating treatment with methadone, $92 \%$ were consultants, and $80 \%$ of these considered themselves to be experienced in prescribing methadone for pain treatment. Seventy-six percent of the physicians started methadone without consulting a colleague. Of those seeking guidance, a palliative medicine specialist was consulted in $64 \%$, a pain specialist in $14 \%$, and other specialists in $22 \%$ of the cases.

\section{Discussion}

In this study with national coverage, we report that methadone is frequently used for the management of complex pain at the end of life in specialized palliative care in Sweden. It is mainly used in low doses as an add-on therapy to an ongoing regular opioid treatment of cancer-related pain, usually with good effect, and without any reported severe adverse effects.
Despite the reported unique properties of methadone, there is only limited scientific evidence that it is effective in managing severe pain due to cancer in adults. ${ }^{10,12,20}$ A Cochrane review from 2017 considers methadone unlikely to have a role as a first-line treatment for cancer-related pain, mainly due to the difficulties around dose titration and the risks of severe adverse effects. ${ }^{11}$ Also, a review from Fallon and Laird gives only a weak recommendation to support opioid combination therapy. ${ }^{21}$ It was interesting, therefore, to find that as many as $8.6 \%$ of the patients enrolled in specialized palliative care in Sweden were successfully initiated on methadone.

In patients with a poor analgesic response after opioid dose escalation, an addition of low doses of methadone to improve analgesia is reported effective. ${ }^{22}$ Recent studies have reported that $50 \%-80 \%$ of patients with cancer-related pain have improved pain control after the addition of methadone, most likely benefitting from methadone's NMDA receptorinhibiting properties. ${ }^{14,23,24}$ Indirectly, in most of our patients, this was supported by the observation that an increase of the primary strong opioid (with mu-receptor effect) did not improve pain control, whereas methadone did. However, a possible, limited synergistic mu-receptor effect cannot be outruled, as laboratory research has reported a synergistic effect when combining methadone with morphine or diamorphine, whereas there is only an additive effect when combining with oxycodone or fentanyl. ${ }^{25}$ In our study, despite morphine being considered one of the first-line opioids, ${ }^{9}$ morphine was used in only $11 \%$ of the patients. Most patients were prescribed fentanyl $(50 \%)$ or oxycodone $(32 \%)$.

The use of low-dose methadone, in addition to a regular ongoing opioid therapy for the treatment of complex pain in advanced cancer, is a promising, but not yet evidence-based approach. However, the prevalence of, and the more specified indications for the use of, low-dose methadone has not been, to the best of our knowledge, previously described. In this study, we report that in Swedish specialized palliative care, methadone was used in close to one-tenth of the patients, almost exclusively (96\%) as an add-on low dose to other opioids and usually due to poor pain control (74\%) at the end of life.

Complex pain is often due to a combination of nociceptive and neuropathic pain mechanisms and management often remains a challenge. ${ }^{3,26,27}$ Therefore, the presence of complex, mixed nociceptive and neuropathic pain in $70 \%$ of the patients in our study was not surprising. It is interesting to note that methadone was also initiated as a primary add-on 
therapy against neuropathic pain in $17 \%$ of the patients. The setup of the study did not allow us to explore whether these patients also received traditional antineuropathic pharmacological treatment.

In specialized palliative home care in Sweden, patients administer their own drugs and usually have visits from the staff only once or twice a week. This is of importance since there are concerns about safety in ambulatory settings due to the inherent risk of respiratory depression and QTc prolongation associated with methadone use. ${ }^{28}$ In our material, patients in home care settings were safely initiated on methadone. Our findings are corroborated by two other studies. Porta-Sales et al. and Hawley et al. described safe and efficacious conversions from regular opioids to methadone in outpatient palliative cancer clinic settings, using either a stop-opioid-and-go-methadone approach or a start-low goslow switch to methadone, over up to 18 days. ${ }^{29,30}$ Weschules et al. explored the frequency and the utilization patterns of methadone on 21,219 hospice patients in a home care setting in Philadelphia in 2003, and found that methadone accounted for $1.7 \%$ of all long-acting opioid prescriptions. ${ }^{31}$

The reported adverse effects were expected and manageable mu-receptor-related side effects. Only $2 \%$ of the patients discontinued methadone treatment because of adverse effects. Furthermore, the proportion of patients with sedation and delirium was lower than in earlier reports. ${ }^{14,24}$

Twenty-eight percent of the patients received methadone parenterally from the start, almost exclusively through the subcutaneous (SC) route; only a few received a continuous intravenous infusion (1.2\%). During the last 24 hours with methadone, the proportion of patients with parenteral administration was $50 \%$, but methadone in continuous intravenous infusion was still uncommon.

SC continuous methadone administration has been associated with erythema. ${ }^{32}$ However, if the infusion site is rotated every one or two days or if methadone is diluted, these reactions tend to be mild and manageable. ${ }^{13,33-35}$ Our findings indicate that the use of SC methadone injections is both common and practically manageable, thereby providing health care personnel with a useful tool for preventing complex pain at end of life.

In our study, $86 \%$ of the patients initiated on methadone stayed on the therapy until death, after a median of 21 days. During the same period, the total median MEDD of concomitant opioids did not increase more than $8 \%$, despite these patients being at the end of life and suffering from complex cancer-related pain. This is consistent with previous findings where the addition of methadone to an ongoing opioid therapy was associated with lower escalation rates or even decreased doses of regular opioids. At the same time, pain was significantly improved or maintained at acceptable levels. ${ }^{14,23,24,36}$

We recognize some limitations of our study. Voluntary participation by the specialized palliative care units possibly resulted in the selection of units already experienced with methadone, presumably with an inherited positive attitude. Moreover, adherence to agreed routines for data collection is a common problem for most registers, and SRPC constitutes no exception. ${ }^{37}$ Validated instruments for pain and symptom assessments were used to a large extent, but, unfortunately, these are still not routinely used in every unit. When not used, the reporting was based on subjective judgments by the physician or nurse performing the registration. Consequently, symptom assessments to some degree depended on the level of knowledge, skills, and personal attitudes. With $45 \%$ of invited units participating in our study, we cannot claim the results to be representative of all specialized palliative care in Sweden but, nevertheless, they describe current national practice. Strengths include the widespread use of the SRPC in Sweden and the geographical representation from almost all parts of the country, enhancing the external validity of our findings.

\section{Conclusion}

The addition of low-dose methadone as a coanalgesic to another ongoing opioid therapy appears to be a safe way to benefit from the unique pharmacodynamics of methadone, especially in patients with complex cancer-related pain at the end of life. In Sweden, this regimen already appears to constitute a well-established part of the therapeutic arsenal in specialized palliative home care, in the hands of predominately experienced physicians.

There is a need for further studies. Appropriately designed randomized controlled trials should confirm the efficacy of the low-dose add-on methadone approach.

\section{Acknowledgments}

The authors wish to thank Greger Fransson and the units in the SRPC for their valuable participation with data collection and Sara Runesdotter for her assistance with the statistics. David Boniface is acknowledged for the linguistic revision.

\section{Funding Information}

This work was supported by the Stockholm Sjukhem Foundation and by the Stockholm County Council (ALF project).

\section{Author Disclosure Statement}

The authors declare that they have no conflicts of interest.

\section{References}

1. van den Beuken-van Everdingen MH, Hochstenbach LM, Joosten EA, et al.: Update on prevalence of pain in patients with cancer: Systematic review and meta-analysis. J Pain Symptom Manage 2016;51:1070-1090.e1079.

2. Greco MT, Roberto A, Corli O, et al.: Quality of cancer pain management: An update of a systematic review of undertreatment of patients with cancer. J Clin Oncol 2014; 32:4149-4154.

3. Finnerup NB, Attal N, Haroutounian S, et al.: Pharmacotherapy for neuropathic pain in adults: A systematic review and meta-analysis. Lancet Neurol 2015;14:162-173.

4. Sotgiu ML, Valente M, Storchi R, et al.: Cooperative N-methyl-D-aspartate (NMDA) receptor antagonism and mu-opioid receptor agonism mediate the methadone inhibition of the spinal neuron pain-related hyperactivity in a rat model of neuropathic pain. Pharmacol Res 2009;60: 284-290.

5. Davis MP, Walsh D: Methadone for relief of cancer pain: A review of pharmacokinetics, pharmacodynamics, drug interactions and protocols of administration. Support Care Cancer 2001;9:73-83. 
6. Lugo RA, Satterfield KL, Kern SE: Pharmacokinetics of methadone. J Pain Palliat Care Pharmacother 2005;19: 13-24.

7. Nilsson MI, Meresaar U, Anggard E: Clinical pharmacokinetics of methadone. Acta Anaesthesiol Scand Suppl 1982;74:66-69.

8. Fredheim OM, Moksnes K, Borchgrevink PC, et al.: Clinical pharmacology of methadone for pain. Acta Anaesthesiol Scand 2008;52:879-889.

9. Caraceni A, Hanks G, Kaasa S, et al.: Use of opioid analgesics in the treatment of cancer pain: Evidence-based recommendations from the EAPC. Lancet Oncol 2012;13:e58-e68.

10. Bruera E, Palmer JL, Bosnjak S, et al.: Methadone versus morphine as a first-line strong opioid for cancer pain: A randomized, double-blind study. J Clin Oncol 2004;22:185-192.

11. Nicholson AB, Watson GR, Derry S, et al.: Methadone for cancer pain. Cochrane Database Syst Rev 2017:2;Cd003971.

12. Mercadante S, Casuccio A, Agnello A, et al.: Morphine versus methadone in the pain treatment of advanced-cancer patients followed up at home. J Clin Oncol 1998;16:36563661.

13. Hawley P, Wing P, Nayar S: Methadone for pain: What to do when the oral route is not available. J Pain Symptom Manage 2015;49:e4-e6.

14. Furst P, Lundstrom S, Klepstad P, et al.: Improved pain control in terminally ill cancer patients by introducing low-dose oral methadone in addition to ongoing opioid treatment. J Palliat Med 2018;21:177-181.

15. Lundstrom S, Axelsson B, Heedman PA, et al.: Developing a national quality register in end-of-life care: The Swedish experience. Palliat Med 2012;26:313-321.

16. Swedish Register for Palliative Care: Annual Report 2017. www.palliativ.se (last accessed January 1, 2019).

17. Fisch M: Cancer pain dosing strategies for oral methadone. In: Fisch MJ (ed): Cancer Pain Management. New York: McGraw Hill Medical, 2007, pp. 307-310.

18. Bruera E, Kuehn N, Miller MJ, et al.: The Edmonton Symptom Assessment System (ESAS): A simple method for the assessment of palliative care patients. J Palliat Care 1991;7:6-9.

19. Schildmann EK, Groeneveld EI, Denzel J, et al.: Discovering the hidden benefits of cognitive interviewing in two languages: The first phase of a validation study of the Integrated Palliative care Outcome Scale. Palliat Med 2016;30:599-610.

20. Mercadante S, Porzio G, Ferrera P, et al.: Sustained-release oral morphine versus transdermal fentanyl and oral methadone in cancer pain management. Eur J Pain 2008;12: 1040-1046.

21. Fallon MT, Laird BJ: A systematic review of combination step III opioid therapy in cancer pain: An EPCRC opioid guideline project. Palliat Med 2011;25:597-603.

22. Mercadante $\mathrm{S}$ : Opioid combination for cancer pain. $\mathrm{Br} \mathbf{J}$ Cancer 2004;90:2049.

23. Wallace E, Ridley J, Bryson J, et al.: Addition of methadone to another opioid in the management of moderate to severe cancer pain: A case series. J Palliat Med 2013;16: 305-309.
24. Courtemanche F, Dao D, Gagne F, et al.: Methadone as a coanalgesic for palliative care cancer patients. J Palliat Med 2016;19:972-978.

25. Pasternak GW: Preclinical pharmacology and opioid combinations. Pain Med 2012;13(Suppl 1):S4-S11.

26. Portenoy RK, Foley KM, Inturrisi CE: The nature of opioid responsiveness and its implications for neuropathic pain: New hypotheses derived from studies of opioid infusions. Pain 1990;43:273-286.

27. Rayment C, Hjermstad MJ, Aass N, et al.: Neuropathic cancer pain: Prevalence, severity, analgesics and impact from the European Palliative Care Research CollaborativeComputerised Symptom Assessment study. Palliat Med 2013;27:714-721.

28. Lovell AG, Protus BM, Saphire ML, et al.: Evaluation of QTc interval prolongation among patients with cancer using enteral methadone. Am J Hosp Palliat Care 2019;36: 177-184.

29. Porta-Sales J, Garzon-Rodriguez C, Villavicencio-Chavez C, et al.: Efficacy and safety of methadone as a second-line opioid for cancer pain in an outpatient clinic: A prospective open-label study. Oncologist 2016;21:981-987.

30. Hawley P, Chow L, Fyles G, et al.: Clinical outcomes of start-low, go-slow methadone initiation for cancer-related pain: What's the hurry? J Palliat Med 2017;20:1244-1251.

31. Weschules DJ, McMath JA, Gallagher R, et al.: Methadone and the hospice patient: Prescribing trends in the home-care setting. Pain Med 2003;4:269-276.

32. Bruera E, Fainsinger R, Moore M, et al.: Local toxicity with subcutaneous methadone. Experience of two centers. Pain 1991;45:141-143.

33. Elsass K, Marks S, Malone N: Nonoral routes of methadone for analgesia in palliative care \#358. J Palliat Med 2018;21: 1357-1358.

34. Mathew P, Storey P: Subcutaneous methadone in terminally ill patients: Manageable local toxicity. J Pain Symptom Manage 1999;18:49-52.

35. Makin MK: Subcutaneous methadone in terminally-ill patients. J Pain Symptom Manage 2000;19:237-238.

36. Mercadante S, Villari P, Ferrera P, et al.: Addition of a second opioid may improve opioid response in cancer pain: Preliminary data. Support Care Cancer 2004;12: 762-766.

37. Martinsson L, Heedman PA, Lundstrom S, et al.: Validation study of an end-of-life questionnaire from the Swedish Register of Palliative Care. Acta Oncol 2011;50: 642-647.
Address correspondence to:

Per Fürst, $M D$

Palliative Medicine

Stockholms Sjukhem Foundation

Box 12230

SE-10226 Stockholm Sweden

E-mail: per.furst@ki.se 


\section{Appendix 1. The Online Methadone Add-On Survey Questionnaire}

1. What was the rationale for the prescription of methadone to this patient?

2. What was the main pain mechanism?

3. What was the responsible physician's highest level of education?

4. How experienced in the use of methadone was the responsible physician?

5. If the responsible physician consulted other physicians regarding the prescription of methadone, who was consulted?

6. Methadone treatment:

Duration of treatment, doses, routes, and number of daily administrations at start and during the final 24 hours

How many adjustments were made to the methadone dose during the treatment period?
7. Date and reason for cessation of methadone treatment (including death)

8. In case of concomitant opioid use, Which opioid was used?

What was the daily regular dose, as-needed doses at start and during the final 24 hours, and route of administration?

9. In this patient, how do you appraise the overall analgesic effect of methadone?

10. Were there any adverse effects associated with methadone? Which ones?

11. Altogether, was the introduction of methadone of benefit to this patient? 